\title{
Adnexal torsion-symptoms, diagnosis and management: a review of literature
}

\author{
Nidhi Jain*, Rahul Manchanda, Sravani Chithra, Anshika Lekhi
}

Manchanda's Endoscopic Centre, New Delhi, India

Received: 04 April 2016

Accepted: 25 April 2016

\section{*Correspondence:}

Dr. Nidhi Jain,

E-mail: nidhijain270587@gmail.com

Copyright: () the author(s), publisher and licensee Medip Academy. This is an open-access article distributed under the terms of the Creative Commons Attribution Non-Commercial License, which permits unrestricted non-commercial use, distribution, and reproduction in any medium, provided the original work is properly cited.

\begin{abstract}
Adnexal torsion accounts for $3 \%$ of all gynaecologic emergencies. It consists of spectrum of pathologies including ovarian torsion, ovarian torsion with tubal torsion and isolated fallopian tube torsion. It usually presents with acute abdominal pain along with gastrointestinal symptoms such as nausea and vomiting. But due to presence of nonspecific clinical features, diagnosis of adnexal torsion is a difficult task which requires high degree of clinical suspicion. Ultrasound with Doppler is helpful in its diagnosis. But even Doppler studies can miss the cases of adnexal torsion. So, laparoscopy is the gold standard for its diagnostic evaluation and simultaneous management. Conservative surgery should be preferred to preserve the future reproductive function. But in some cases such as infarcted ovaries, older women and suspicion of malignancy, adnexa may be required to be removed. Thus, prompt diagnosis and timely management of adnexal torsion is required to prevent the irreversible damage to adnexa. The authors here present a review of literature concerning the clinical features, diagnosis and management of adnexal torsion via a case series of six cases.
\end{abstract}

Keywords: Adnexal torsion, Isolated fallopian tube torsion, Ovarian torsion

\section{INTRODUCTION}

Adnexal torsion is one of the most common gynaecological emergency conditions to be misdiagnosed by the clinicians. It consists of spectrum of pathologies including ovarian torsion, ovarian torsion with tubal torsion and isolated fallopian tube torsion (IFTT). It accounts for $3 \%$ of all gynaecologic emergencies. ${ }^{1}$

It is most commonly seen in women of reproductive age group. However, no age is exempt from this pathology and cases have been reported in both prepubertal girls and postmenopausal women. The most common presenting features are acute onset of pelvic pain which may be fluctuating or radiating to loin, with nausea and vomiting. Low-grade pyrexia may also be present. Signs include tachycardia, rebound tenderness and adnexal mass. Prompt intervention is necessary to preserve the reproductive function. Laparoscopic detorsion is considered as the procedure of choice. However, in older women, in case of gangrenous tube/ovary, and in suspicion of malignancy, salpingoopherectomy need to be performed.

With this background, authors here present a review of literature to discuss clinical presentation, diagnosis and management of adnexal torsion via a case series of six cases.

\section{CASE SUMMARY}

Case 1

An 11-year-old prepubertal girl presented to emergency with acute abdominal pain in the right lower quadrant, which was piercing, non-radiating and associated with nausea and vomiting. On examination, tenderness was present in right iliac fossa. Her blood investigations were 
normal. On ultrasound, large anechoic cystic lesion of size $6.8 \times 6.6 \times 4.9 \mathrm{~cm}$ was visualized in right adnexa. Right ovary was seen adjacent to cyst and left side ovary and tube were normal. MRI showed a well-defined rounded cystic lesion of size $5.2 \times 5.5 \times 7.1 \mathrm{~cm}$, extending from supravesical area to right adnexa, separated from ovary. No internal septations, calcifications or mural nodule was seen within the lesion. Uterus and left side adnexa were normal. CA-125 was normal (13.24 U/mL). Diagnostic laparoscopy was done and patient was found to have an IFTT on right side, twisting three times with huge paratubal cyst of size $8 \times 6$ $\mathrm{cm}$. Right sided ovary was normal. Detorsion of tube followed by cystectomy was done. The postoperative period was uneventful.

\section{Case 2}

13-year-old girl came to casualty due to acute pain in the right lower abdomen lasting for two days. Her last period was twenty days prior to admission. She was not sexually active. On physical examination, abdominal guarding and tenderness was present. Her laboratory investigations were normal. Ultrasound examination revealed a right adnexal cyst of size $5 \times 6 \mathrm{~cm}$ without internal septations or calcifications. The acute onset of pain, physical finding, and detection of an adnexal mass on ultrasonography raised suspicion of right adnexal torsion. It was decided that the patient should undergo urgent laparoscopy, which was performed. On laparoscopy, right sided IFTT (Figure 1A) was found with bilateral paratubal cyst, of size $7 \mathrm{~cm}$ on right side (Figure 1B) and $3 \mathrm{~cm}$ on left side along with normal ovary (Figure 1C). Following surgery, patient was well and discharged on next day of surgery.

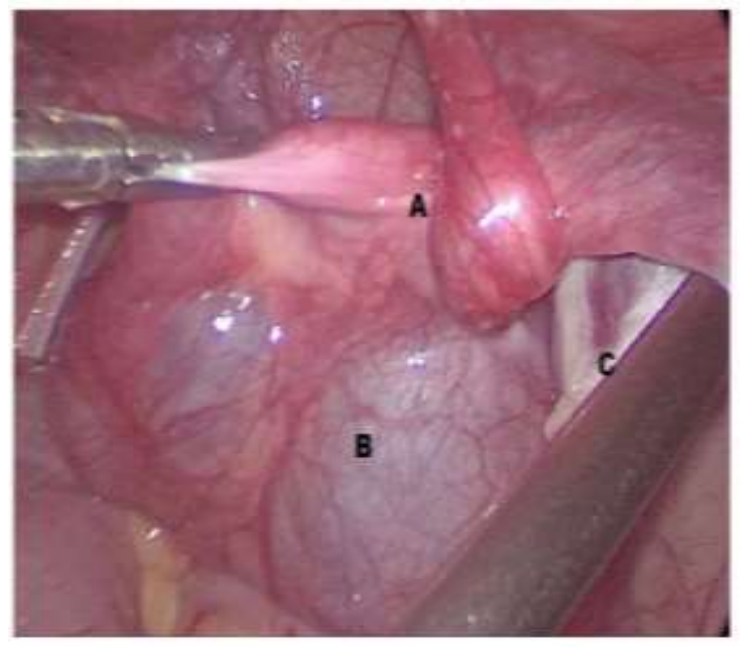

Figure 1: A-Right isolated fallopian tube torsion, B -Right paratubal cyst, C- Right normal ovary.

\section{Case 3}

20 years old unmarried girl presented to casualty with acute pain in abdomen since 3 days. Pain was intermittent, colicky in nature, non-radiating and associated with nausea and vomiting. Abdominal tenderness was present in right iliac fossa on palpation. On per rectal examination, a cystic tender mass was palpated in posterior fornix. Blood investigations were normal. On sonography, a large anechoic cyst of size $6 \times$ $5 \mathrm{~cm}$ was seen in right adnexa, suspected to be ovarian origin, with maintained vascular flow in ovarian pedicle on Doppler studies. Serum markers were normal. Urine pregnancy test was negative. On laparoscopy, surprisingly we found isolated right side tubal torsion with 4 twists and bilateral ovaries were normal. Tube was dilated and gangrenous in appearance. Right hydrosaplinx was seen at fimbrial end. Detorsion was done, but tube remain black, gangrenous and necrotic, hence decision for laparoscopic salpingectomy was taken. Post-operative period was uneventful.

\section{Case 4}

9 years old premenarchal girl was having intermittent, piercing pain in lower abdomen on left side since 2 days for which she was being treated by a paediatrician. Ultrasound showed normal uterus, tubes and ovaries. Mild free fluid was present in pelvis. She was treated conservatively with antibiotics. But since, pain did not subsided so she was referred to us. On examination, abdominal distension along with guarding and tenderness was present. With strong suspicion of torsion, decision of emergency laparoscopy was taken. On laparoscopy, haemoperitoneum of around $500 \mathrm{ml}$ was found. Left side adnexa were twisted (Figure 2A). Adnexa were black and gangrenous in appearance. Left ovarian cyst of size of $5 \times$ $6 \mathrm{~cm}$ (Figure 2B) was also seen. Detorsion was done but blood supply could not be restored (Figure 3). So, unfortunately left sided salpingoopherectomy needed to be done. Postoperatively she was well and discharged the day after surgery.

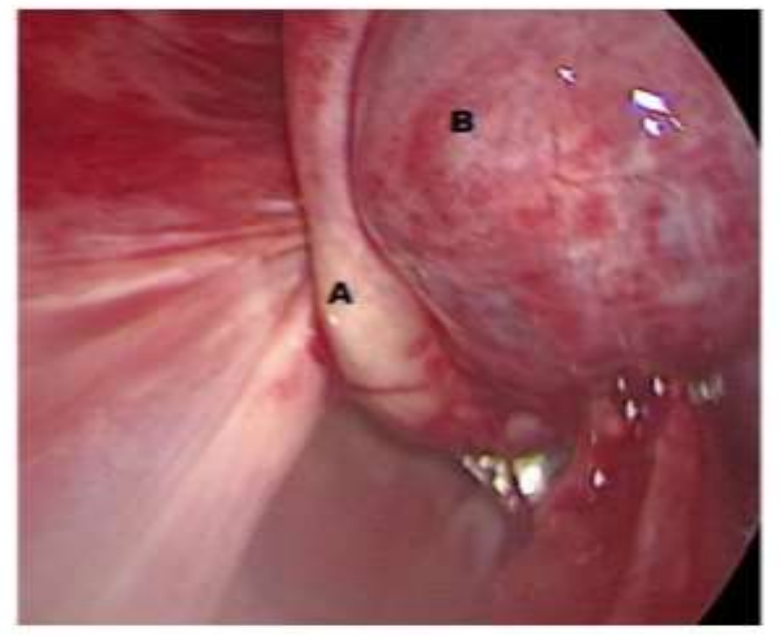

Figure 2: A-Left adnexal torsion, B-Left ovarian cyst. 


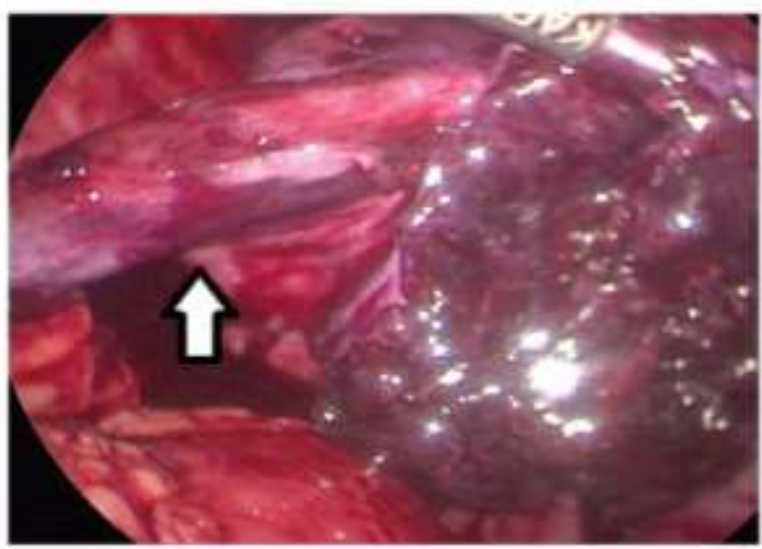

Figure 3: Arrow: Non restoration of vascularity even after detorsion.

\section{Case 5}

18 years unmarried girl presented to our emergency with pain in right iliac fossa since one day. Pain was intermittent and dull aching in nature. She had 2-3 episodes of non-bilious vomiting in last 24 hours. She had similar episode of acute pain five month back, which was managed conservatively. She was a known case of polycystic ovarian disease and on oral contraceptive pills since 3 months. On examination, rebound tenderness was present in right iliac fossa. In blood investigations, leukocytosis was found. CA-125 was normal. On Sonography, right ovary was enlarged with a thick walled, clear cyst of size $7.1 \times 5.7 \times 6.5 \mathrm{~cm}$. Left ovary was also enlarged with peripherally arranged follicles and a simple cyst of size $2.8 \mathrm{~cm}$. On colour Doppler, blood flow in both ovarian pedicles was maintained.

Considering the recurrent episode of acute pain and tenderness on examination, authors decided to take up the patient for diagnostic laparoscopy. Per-operatively, she was found to have right adnexal torsion, three and half turns with a right ovarian cyst. Detorsion of adnexa with right cystectomy was done. Left ovary was also enlarged with multiple follicles and a haemorrhagic cyst of $3 \times 2$ $\mathrm{cm}$, which was excised. Patient was well post operatively and discharged the day after surgery.

\section{Case 6}

82 year old postmenopausal woman presented to the gastro-enterology department with complaints of lower abdominal pain and constipation for duration of two weeks. On Per abdomen examination, mild tenderness was present. Bowel sounds were present. Her blood parameters were normal. X-ray abdomen was normal. Colonoscopy revealed diverticulosis of colon $20 \mathrm{~cm}$ from anal verge. CT abdomen was done which revealed diverticulosis of colon along with a right adnexal mass of size $7 \times 6 \mathrm{cms}$ with heterogeneous density, which was inseparable from ovary. Diagnosis of dermoid cyst with diverticulitis was made. Patient was referred to the gynaecology department. Bimanual examination revealed tenderness and fullness in the right fornix. CA-125 was normal. A diagnostic and operative laparoscopy was done. Pus was seen in the abdominal cavity up to right sub-diaphragmatic area which was aspirated. Bowel was distended and vision was obstructed. No breach in bowel was seen. Inflammation was present. Right ovarian dermoid cyst of size $12 \times 10 \mathrm{~cm}$ (Figure 4) was seen which had undergone torsion three and a half times (Figure 5). Right oopherectomy was done and sent for histopathology, which confirmed the diagnosis of dermoid cyst.

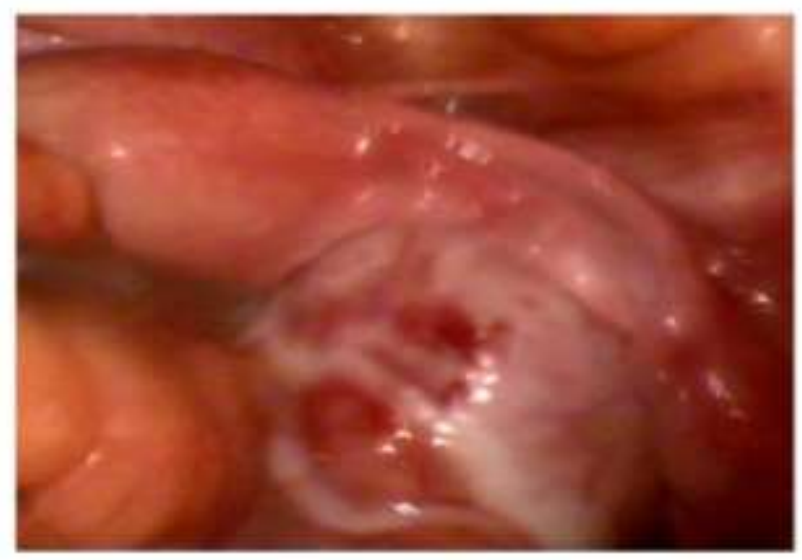

Figure 4: Right ovarian dermoid cyst.

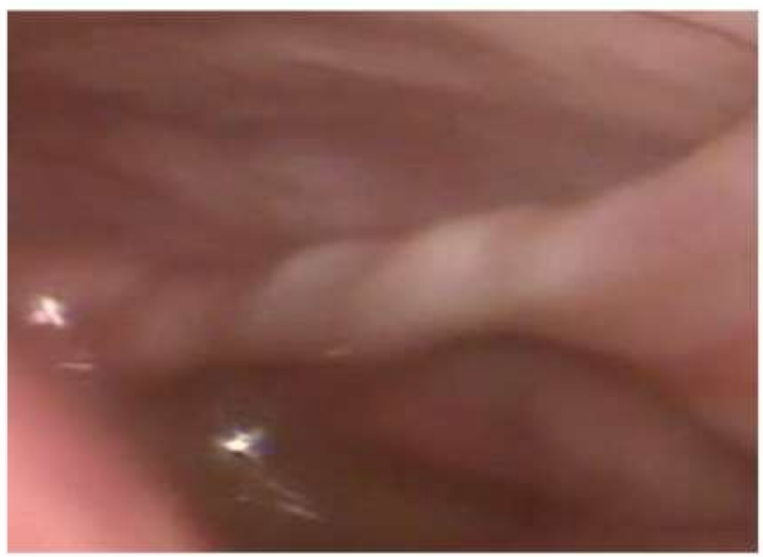

Figure 5: Right adnexal torsion with three and half twists.

\section{DISCUSSION}

Adnexal torsion includes torsion of the ovary, tube or both. It is estimated to be responsible for only a small number of all gynaecological emergencies, but is a common diagnostic challenge in the emergency setting. Its diagnosis is difficult and is mainly based on clinical symptoms, examination findings and imaging modalities. Here we discuss the literature regarding its presentation, diagnosis and management. 


\section{Isolated fallopian tube torsion}

Isolated fallopian tube torsion is defined as the twisting of the fallopian tube by at least one complete turnaround a centre-line consisting of the infundibulopelvic ligament or tubo-ovarian ligament. ${ }^{2}$ It is an uncommon cause of acute lower abdominal pain with reported incidence as $1: 1500000 .^{3}$

Bland-Sutton T. ${ }^{4}$ has described the first case of IFTT in 1890 as a rare cause of lower abdominal pain and since then several hundred cases have been reported in the literature.

It primarily affects women of reproductive age group. However, it can be seen even in prepubertal girls and postmenopausal women. In 2013, Casey RK et al ${ }^{5}$ did a retrospective study to review fifteen cases of IFTT in paediatric age group, in which youngest child who was diagnosed with IFTT was 8 years old. In a case report by Djing et $\mathrm{al}^{6}$ in 2007, a 70 years old woman was diagnosed with IFTT. Here, we have seen IFTT in a 9 years old premenarchal girl, 13 years old girl who has attained her menarche and a 20 years old unmarried girl.

The aetiology of IFTT is not well known. Several risk factors have been identified, which are divided into 2 groups: intrinsic and extrinsic (Table 1). Tubal torsion is defined as primary when it is not associated with any of these risk factors, being idiopathic in aetiology and secondary if associated with underlying pathology.

Table 1: Risk factors associated with isolated fallopian tube torsion.

\begin{tabular}{|ll|}
\hline Intrinsic factors & Extrinsic factors \\
\hline $\begin{array}{l}\text { Ovarian factors: } \\
\text { Congenital factors: }\end{array}$ & $\begin{array}{l}\text { Polycystic ovarian disease } \\
\text { Ovarian mass } \\
\text { Para ovarian mass } \\
\text { Ovarian hyper stimulation } \\
\text { syndrome }\end{array}$ \\
\hline $\begin{array}{l}\text { Acquired factors: } \\
\text { Hydrosaplinx/hemosalpinx } \\
\text { Tubal ligation }\end{array}$ & Uterine factors: \\
Endometriosis & Mass \\
Tubal neoplasm & Pregnancy \\
Abnormal tubal peristalsis & \\
\hline & Miscellaneous: \\
& Pelvic inflammatory \\
& disease \\
& Pelvic congestion of \\
& mesosalpinx \\
& Trauma \\
& Sudden body position \\
& changes \\
\hline
\end{tabular}

In 2012, Gaied et $\mathrm{al}^{3}$ did a review of literature of 20 years data for IFTT in children and found that, secondary tubal torsion was found in $44 \%$ of cases associated with underlying tubal pathology while rest $56 \%$ were primary fallopian tube torsion, being idiopathic in origin. While in another study in 2013 by Casey RK et $\mathrm{al}^{4}$, secondary IFTT was more commonly found $(53 \%)$ than primary IFTT $(47 \%)$.

All three cases presented here were secondary in origin being associated with paratubal cyst in first two cases and with hydrosaplinx in the third case.

In 2013, Kardakis $\mathrm{S}$. et $\mathrm{al}^{7}$ suggested the sequential mechanism of its pathogenesis that involves mechanical obstruction of adnexal veins and lymphatics, leading to pelvic congestion and edema with subsequent enlargement of the fimbrial end, which in turns leads to partial to complete torsion of the involved tube. Vascular supply to the fallopian tubes comes from both ovarian and uterine vessels, resulting in the possibility of isolated tubal torsion without vascular compromise of the ovary. Furthermore, distal obstruction of fimbrial end along with continuous secretion of tubal glands leads to formation of hydrosaplinx, which can also lead to tubal torsion.

IFTT is usually unilateral and more frequently diagnosed on the right side. In our study also, in all three cases we found IFTT on right side. The possible explanations for this are presence of sigmoid colon on left side which prevent torsion of that side. Secondly, right sided pathology is more commonly evaluated surgically with appendicitis as differential diagnosis. Another reason is the variance in venous return flow rate between the right and left fallopian tubes. However, in 2010, Wong et $\mathrm{al}^{8}$ reported case series of six cases of IFTT in which five women were found to have torsion of left side. While in a case reported by Lima et $\mathrm{al}^{9}$ in 2011 , a 13 years old girl was found to have torsion of both sided tubes simultaneously.

The clinical presentation of IFTT is non-specific and hence a challenge for the clinician to recognize and differentiate it from multiple other aetiologies (Table 2). Most of the cases present with acute abdomen with nausea and vomiting. Low grade pyrexia may also be seen. In our series also, all three girls presented with acute abdominal pain.

Table 2: Differential diagnosis of acute pain in lower abdomen.

\begin{tabular}{|ll|}
\hline $\mathbf{1}$ & Appendicitis \\
\hline $\mathbf{2}$ & PID \\
\hline $\mathbf{3}$ & Functional ovarian cyst \\
\hline $\mathbf{4}$ & Ectopic pregnancy \\
\hline $\mathbf{5}$ & Renal colic \\
\hline $\mathbf{6}$ & Fibroid: torsion, red degeneration, haemorrhage \\
\hline $\mathbf{7}$ & Ruptured ovarian cyst \\
\hline $\mathbf{8}$ & Diverticulitis \\
\hline $\mathbf{9}$ & OHSS \\
\hline
\end{tabular}


However, in case report by Philips et al ${ }^{10}$ in 2009,34 years old woman who presented with chronic lower abdominal pain, was found to have isolated right sided tubal torsion. On clinical examination, abdominal tenderness is usually found with or without peritoneal signs. Laboratory findings are usually normal, as seen in our patients also. Necrosis can cause leukocytosis, raised sedimentation rate and $\mathrm{C}$-reactive protein.

Ultrasound is often the first imaging modality done in a case of acute abdominal pain. The sonographic findings are of IFTT are dilated tube with thickened echogenic walls, normal-appearing uterus and ovaries with normal blood flow and free fluid in pouch of Douglas. These features are seen in most of the cases of IFTT but they are not pathognomic for the diagnosis. ${ }^{11}$

In 2009 Vijaya Raghavan SB et $\mathrm{al}^{12}$ has described the sonographic whirlpool sign as the specific sign of fallopian tube torsion. It is described as the presence of a whirlpool mass in close proximity to the fallopian tube. This mass is usually not as big or obvious compared with that seen in ovarian torsion; hence, a rocking movement of the endovaginal probe is made over this area. However, this sign is difficult to elicit in most of the patients.

It is important to differentiate between IFTT and total adnexal torsion for which the use of colour Doppler ultrasound is required. Colour Doppler demonstrates normal ovarian blood flow and high-impedance flow with reversed diastolic or absent flow in the wall of the dilated tube or tubal mass. But a normal Doppler blood flow study cannot rule out the diagnosis of IFTT because of the dual vascular supply of fallopian tubes.

A study was done by Sun LT et $\mathrm{al}^{13}$ in 2014 , to determine role of ultrasound for diagnosis of IFTT. Retrospective data was collected and 11 cases of IFTT were found. Of these 11 cases, only $4(36.4 \%)$ cases were diagnosed accurately by imaging modalities preoperatively. Similarly, in our study in case 3, Doppler studies showed normal blood flow but later on, when laparoscopy was done, patient was diagnosed with IFTT.

Delayed presentation, non-specific features and misdiagnosis of IFTT can lead to various complications such as fallopian tube necrosis and gangrenous transformation, leading to an increased risk for super infection and peritonitis. Local necrosis can also result in irreversible damage to the ipsilateral ovary. ${ }^{14}$ To prevent this complication, prompt diagnosis of torsion and management is essential.

As the time duration between onset of pain and surgery increases, chances of saving the tubes decreases. In 2008, Harmon et $\mathrm{al}^{15}$ conducted a retrospective study of 8 IFTT cases and found the mean time taken from initial imaging to surgery was 1.67 days, because of which tube could not be salvaged in any of the cases. Here also we were not able to save the tube in one of the case (case 3) probably due to delayed presentation (3 days).

Laparoscopy is the now recommended as the gold standard in the management of fallopian tube torsion. It offers an added advantage of not only diagnosing the pathology but also simultaneous detorsion of adnexa to re-establish the blood supply so as to salvage the tube.

Cohen $\mathrm{S}$ et $\mathrm{al}^{16}$ has done a study in 2003 to compare the role of laparoscopy to laparotomy for management of tubal torsion. They found that laparoscopy is the most specific diagnostic tool for evaluating torsion and its simultaneous management. Laparoscopy is preferred over laparotomy since recovery after laparoscopy is faster, and it causes fewer pelvic adhesions, which is particularly important for women of reproductive age, who wish to preserve their fertility.

Treatment options of the isolated tubal torsion include either detorsion or salpingectomy, depending on the timing of surgical intervention and presence of complications.

In a retrospective study by Boukaidi SA et $\mathrm{al}^{17}$ in 2011 , out of 13 cases of IFTT, in 9 cases $(69 \%)$ salpingectomy was done. On histopathology, resected tubes showed presence of ciliated cells with moderate ischemic infarction in $50 \%$ of cases. Salpingoneostomy was also reported as a therapeutic approach, and one case was treated with this modality with an uneventful follow-up of 26 months.

Similarly in 2012 Gaied et $\mathrm{al}^{3}$ did a study and found that salpingectomy was done in $88 \%$ of patient and only $12 \%$ of patients could be managed by detorsion alone. They concluded that tubal conservation should be favoured if possible but due to delay in diagnosis, it is often difficult to salvage the tubes. Here we have managed two cases conservatively while in third case we did salpingectomy.

\section{Ovarian torsion}

Ovarian torsion (OT) is the fifth most common problem requiring emergency surgery in gynaecology. It is defined as the twisting of the ovary on its vascular pedicle. If remains undiagnosed, blood supply becomes compromised resulting in tissue necrosis and thus loss of ovarian function. Hence, a quick and confident diagnosis is required to save the adnexal structures from infarction.

Ovarian torsion is most frequently seen in young women of age 20-30 years. In 2005, White $\mathrm{M}$ et al ${ }^{18}$ conducted a retrospective study over a period of 10 years and found fifty two cases of surgically proven ovarian torsion. The mean age of presentation was 33.5 years with interquartile range from 28.7 years to 39.3 years.

Similarly, in another retrospective study by Sahlu $\mathrm{Z}$ et $\mathrm{al}^{19}$ in 2014 , it was found that out of 53 women with 
adnexal torsion, maximum number of women $(62 \%)$ was of age group 20-29 years.

However, ovarian torsion can occur at any age. Its incidence in children has been reported as 4.9 per $100,000 .{ }^{13}$ In 2012, Matthew F Ryan et $\mathrm{al}^{20}$ has reported a case of ovarian torsion in a 5 year old child.

In 2013, Naveen Poonai et $\mathrm{al}^{21}$ performed a retrospective review of cases of ovarian torsion in paediatric population. They analyzed 13 patients and found a seven month child as their youngest case of ovarian torsion. The age range was from 7 month to 18 years with mean age of 12 years and most cases were either in menarchal or in perimenarchal age group.

Ovarian torsion is also seen in postmenopausal women especially those with an ovarian mass. In 2008, Peng YY et $\mathrm{al}^{22}$ had reported case of torsion of huge ovarian cyst in a 90 year old woman.

In a cross sectional study by Zohreh Yousefi et $\mathrm{al}^{23}$ done in 2015, incidence of ovarian torsion in postmenopausal women was found to be $22.6 \%$ with mean age of presentation as $59 \pm 5.8$ years. Here, we have reported the presentation of ovarian torsion in various age groups, ranging from 9 years, 18 years and to 82 years old woman.

Torsion usually occurs unilaterally with $70 \%$ of cases seen on the right side. The reasons for more common occurrence on right side are longer utero-ovarian ligament on right side and limited space on left side due to presence of the sigmoid colon. ${ }^{24}$

In 2015, Yaakov Melcer et $\mathrm{al}^{25}$ found in their study that out of 199 patients, maximum number of women were found to have right sided torsion (62.5\%) while only $36.5 \%$ of cases had pathology on left side and remaining $1 \%$ were found to have bilateral involvement.

Similarly, in study by Haifa A. Al-Turki ${ }^{26}$ in $2016,63.6 \%$ children were found to have torsion of right side while rest $36.4 \%$ had left sided torsion. However in our study, we had only one case of right side torsion and two cases of left side ovarian torsion.

Ovarian torsion is usually associated with pathologically enlarged adnexa. This irregularly enlarged adnexa act as a fulcrum around which ovaries and duct revolves, thereby leading to ovarian torsion or more commonly affecting both tubes and ovaries, thus causing the adnexal torsion. The various risk factors associated with increased risk of ovarian torsion are listed in table 3. But it might occur even in absence of any underlying pathology.

In 2008 , Rousseau $\mathrm{V}$ et $\mathrm{al}^{27}$ has conducted a retrospective study of 40 cases of ovarian torsion in children. 38 cases were of age less than sixteen years while rest two were neonates. Ultrasound was done in all the patients, which showed ovarian mass in 29 cases $(72.5 \%)$, among which 14 cases were of mature teratoma, 10 were cyst adenoma, 2 cases of functional cysts, 2 cases of malignant neoplasm and 1 case had synchronous mature teratoma with carcinoma ovary.

Table 3: Risk factors associated with ovarian torsion.

\begin{tabular}{|ll|}
\hline 1 & Ovarian causes \\
& Polycystic ovaries \\
& Ovarian stimulation \\
& Follicular cysts \\
& Dermoid cyst \\
& Endometrioma \\
& Serous/mucinous cyst adenoma \\
\hline $\mathbf{2}$ & Tubal factors \\
\hline & Tubal ligation \\
& Paratubal cysts \\
\hline $\mathbf{3}$ & Miscellaneous \\
\hline & Pregnancy \\
& Previous abdominal surgery \\
\hline
\end{tabular}

In 2013, Spinelli et $\mathrm{al}^{28}$ presented a review of literature to discuss the management of ovarian torsion in children and adolescents. It was found that $76.7 \%$ cases were associated with underlying pathology, of which $56.7 \%$ had functional ovarian lesion and $20 \%$ had benign neoplasm while in remaining $23.3 \%$ cases ovarian torsion was found with normal adnexa. In our study also, all three cases were found to have ovarian cyst preoperatively. Also, there was history of polycystic ovaries in case of the 18 year old unmarried girl.

The diagnosis of ovarian torsion is challenging as the clinical parameters are nonspecific. Majority of patients presents with acute abdominal pain. Pain is usually sudden in onset, sharp or stabbing in nature, radiating to back and doesn't get relieved by analgesics. Pain is associated with gastrointestinal symptoms such as nausea and vomiting. Low grade fever may also be present. Abdominal tenderness is usually found on palpation. Adnexal mass may also be felt on examination. Blood investigations are usually normal. However in later stages of inflammation, necrosis can lead to leukocytosis or raised erythrocyte sedimentation rate or C-reactive protein.

In 2005, White $\mathrm{M}$ et $\mathrm{al}^{18}$ conducted a retrospective study over a period of ten years and found fifty two cases of ovarian torsion. The main clinical features of presentation were sudden pain in $83 \%$ cases and nausea and vomiting in $59 \%$ cases. Palpable abdominal mass on examination was found in $62.2 \%$ of cases.

Similarly in another study done by Shadinger et $\mathrm{al}^{29}$ in 2008, 39 cases of ovarian torsion were reviewed retrospectively. All patients presented with chief symptom of abdominal pain (100\%). $85 \%$ patients reported nausea and vomiting, while low grade fever was 
seen in only $18 \%$ cases. Leukocytosis was found in $56 \%$ of cases. So, abdominal pain and vomiting were found to be the most frequent clinical indicators of ovarian torsion. In our study also, all patients (100\%) presented with acute pain in abdomen. Vomiting was found in 2 cases $(66.6 \%)$. However, leukocytosis was found in only one case $(33.3 \%)$.

Ultrasound is usually the first imaging modality to be done in case of acute abdominal pain. The features of ovarian torsion on ultrasound include heterogeneous ovarian stroma, string of pearls sign, and free fluid in the cul de sac. Ovarian enlargement of more than $4 \mathrm{~cm}$ is the most consistent ultrasound feature in ovarian torsion. Doppler analysis of the ovarian arterial and venous waveforms is considered as an accurate tool for the evaluation of ovarian torsion. Doppler findings in torsion can be widely variable ranging from little or no intraovarian venous flow to absent arterial flow and absent or reversed diastolic flow.

In a study by Lee EJ et $\mathrm{al}^{30}$, diagnostic value of ultrasound and Doppler in depiction of ovarian torsion was assessed. It was found that out of 32 patients, 28 cases had twisted vascular pedicle on ultrasonography which shows a diagnostic accuracy of 87\%. All 12 patients who showed no blood flow within the twisted vascular pedicle were found to have necrotic ovaries. Thus it was concluded that identification of the twisted vascular pedicle through ultrasonography is suggestive of ovarian torsion, and Doppler studies could be helpful in predicting the viability of adnexal structures by depicting blood flow within the twisted vascular pedicle.

Ben-Ami M et $\mathrm{al}^{31}$ did another study in 2002 to evaluate effectivity of Doppler examination in ovarian torsion. They found that positive predictive value of Doppler study to predict torsion in the absence of venous flow was 94\% and in cases where Doppler revealed venous flow, chances of finding torsion was very unlikely.

However, studies of Doppler flow patterns in torsion are conflicting. The presence of flow at colour Doppler imaging does not allow exclusion of torsion but instead suggests that the ovary may be viable, especially if flow is present centrally. Absence of flow in the twisted vascular pedicle may indicate that the ovary is not viable.

A study was done by Albayram $\mathrm{F}$ et $\mathrm{al}^{32}$ in 2001 , to determine the spectrum of sonographic findings on ultrasound and colour Doppler sonography in a series of pathologically proven cases of ovarian torsion. Ultrasound revealed complex ovarian masses in $73 \%$ of patients, cystic masses in $20 \%$ and a solid mass in $7 \%$. Free fluid was seen in Cul-de-sac in $87 \%$ of patients. $27 \%$ cases were found to have adnexal neoplasm. Doppler findings were abnormal in $93 \%$ of patients however $7 \%$ patients were found to have normal blood flow. Thus it was concluded that diagnosis of ovarian torsion remains challenging and it cannot be based solely on the absence or presence of flow on Doppler studies.

Here in the presented case series, in first case where 9 years old child presented with pain, an ultrasound was reported as normal tubes and ovaries with mild free fluid in pelvis. On laparoscopy, she was found to have adnexal torsion with left ovarian cyst. In second case of 18 year old girl, on ultrasound, an ovarian cyst was found while on Doppler study, normal blood flow was seen. However, during laparoscopy, right torted ovarian cyst was found. Hence, ultrasound and Doppler studies can neither confirm nor exclude all the cases of ovarian torsion. Thus, direct visualisation by laparoscopy is considered as the gold standard to diagnostic evaluation and management of ovarian torsion.

Traditionally, salpingoophorectomy was recommended as the standard treatment for adnexal torsion due to fear of thromboembolism. But now, conservative treatment that consists of untwisting the adnexa followed by cystectomy or cyst aspiration is the management of choice. ${ }^{24}$

In 2004, Aziz D et al ${ }^{33}$ conducted a retrospective study to compare outcomes of conservative surgery and oopherectomy in management of ovarian torsion. 36 cases of ovarian torsion were reviewed, out of which seventeen underwent detorsion with or without ovarian cystectomy, and 19 had oophorectomy. Median time from presentation to surgery was significantly lower in the detorsion group than the oophorectomy group. Postoperative complications and length of stay were similar between the two groups. Despite the ovary being judged intraoperatively as moderately to severely ischemic in $53 \%$ of detorsion cases, follow-up sonogram or ovarian biopsy available in 14 of the 17 cases showed normal ovary with follicular development in each case. Hence, it was concluded that detorsion should be considered as the procedure of choice for most cases of ovarian torsion in children.

In 2012, Ziv Tsafir et $\mathrm{al}^{34}$ conducted a retrospective study to assess risk factors, clinical findings and mode of diagnosis and management in premenarchal children with surgically verified ovarian torsion. Twenty-two cases of ovarian torsion were identified. The mean age of presentation was 12 years. In 19 cases (86.4\%), conservative management, mainly detorsion with cyst drainage or cystectomy, was performed. Oophoropexy was performed in 3 cases $(13.6 \%)$. It was concluded that detorsion followed by cystectomy is the preferred management option of ovarian torsion and may help in preventing the recurrence.

In 2014, Lasso Betancor CE et $\mathrm{al}^{35}$ did a study to evaluate long term follow up of ovarian torsion patients who were managed by laparoscopic detorsion. Seven cases were included. During the first 6 months, the follow-up ultrasound showed good results. However, the long-term outcome evidenced one involved ovary got atrophied and 
two oophorectomies were done, one was due to recurrent adnexal torsion and other because of ovarian teratoma. On conclusion, Laparoscopic conservative management with untwisting the ovary allows the recovery of even macroscopically nonviable ovaries.

This ovary sparing approach after detorsion of ischemic ovaries is considered safe and effective in both adults and children. Since most of the cases are seen in women of reproductive age group, surgeons should always try to salvage the adnexa. However, in some cases where there is suspicion of malignancy or when women have completed her family, radical surgery should be considered. Here, in 2 cases conservative approach was followed including detorsion with cystectomy. However, in one case, even after detorsion, adnexa remain black and gangrenous due to which salpingoophorectomy was done. This could be because of the delayed presentation.

In older and postmenopausal women, oophorectomy is recommended as the treatment of choice. Zohreh Yousefi et $\mathrm{al}^{23} \mathrm{did}$ a retrospective study on management of ovarian torsion in postmenopausal women. 44 cases were included. All patients underwent ovarian removal, based on their postmenopausal status. Given the possibility of neoplastic changes in post-menopausal patients it was advised that frozen section technique should be available at time of surgery in woman of this age group. Here authors have presented case of twisted ovarian cyst in an 82 year old woman, who was managed laparoscopically and oophorectomy was done.

\section{CONCLUSION}

Diagnosis of adnexal torsion is a difficult task which requires high degree of clinical suspicion. Ultrasound with Doppler is helpful in its diagnosis. Laparoscopy is the gold standard for evaluation and management of adnexal torsion. Conservative management including detorsion with or without cystectomy is the preferred treatment. A complete resection of tube/ovary/both is performed when the tissue is gangrenous or when malignancy is suspected or woman has completed her family. Early diagnosis and timely management of adnexal torsion should be done to preserve the reproductive function.

\section{Funding: No funding sources Conflict of interest: None declared \\ Ethical approval: Not required}

\section{REFERENCES}

1. Kontoravdis A, Chryssikopoulos A, Hassiakos D, Liapis A, Zourlas PA. The diagnostic value of laparoscopy in 2365 patients with acute and chronic pelvic pain. Int J Gynaecol Obstet. 1996;52:243-8.

2. Ahn JH, Chung J, Lee TS. Successful laparoscopic surgery for14-month-old infant with ovarian torsion. Obstet Gynecol Sci. 2014;57(2):160-3.
3. Gaied F, Emil S, Lo A, Baird R, Laberge JM. Laparoscopic treatment of isolated salpingeal torsion in children: case series and a 20-year review of the literature. J Laparoendosc Adv Surg Tech A. 2012;22:941-7.

4. Bland-Sutton T. Salpingitis and some of its effects. Lancet. 1890;2:1146.

5. Casey RK, Damle LF, Gomez-Lobo V. Isolated fallopian tube torsion in pediatric and adolescent females: a retrospective review of 15 cases at a single institution. J Pediatr Adolesc Gynecol. 2013;26(3):189-92.

6. Djing DC, Hsu S, Kao SP. Isolated torsion of hydrosaplinx in a postmenopusal woman. JSLS. 2007;11(2):252-4.

7. Kardakis S, Barranca A, Vitelli A, Amore I, Trento F, Caccia G. Isolated Fallopian Tube Torsion. Case Rep Obstet Gynecol. 2013;2013:479698.

8. Wong SW, Suen SH, Lao T, Chung KH. Isolated fallopian tube torsion: a series of six cases. Acta Obstet Gynecol Scand. 2010;89(10):1354-6.

9. Lima M, Libri M, Aquino A, Gobbi D. Bilateral hydrosaplinx with asynchronous tubal torsion: an exceptional finding in a premenarcheal girl. J Pediatr Surg. 2011;46:e27-9.

10. Phillips K, Fino ME, Kump L, Berkeley A. Chronic isolated fallopian tube torsion. Fertil Steril. 2009;92:394.e1-3.

11. Erikci VS, Hosgor M. Isolated salpingeal torsion in children: a case series and review of the literature. Ulus Travma Acil Cerr Derg. 2014;20(1):75-8.

12. Vijaya Raghavan SB, Senthil S. Isolated torsion of the fallopian tube: the sonographic whirlpool sign. J Ultrasound Med. 2009;28(5):657-62.

13. Sun LT, Ning CP, Guo XJ, Li XY, Liu W, Tian JW. Role of ultrasound in diagnosing isolated torsion of fallopian tube. J Obstet Gynaecol Res. 2014;40(1):208-14.

14. Bernardus RE, Van der Slikke JW, Roex AJ, Dijkhuizen GH, Stolk JG. Torsion of the fallopian tube: some considerations on its etiology. Obstet Gynecol. 1984;64:675-8.

15. Harmon JC, Binkovitz LA, Binkovitz LE. Isolated fallopian tube torsion: sonographic and CT features. Pediatr Radiol. 2008;38:175-9.

16. Cohen S, Wattiez A, Seidman D, Goldenberg M, Admon D, Mashiach S et al. Laparoscopy versus laparotomy for detorsion and sparing of twisted ischemic adnexa. JSLS. 2003;7:295-9.

17. Boukaidi SA, Delotte J, Steyaert H, Valla JS, Sattonet C, Bouaziz J et al. Thirteen cases of isolated tubal torsions associated with hydrosalpinx in children and adolescents, proposal for conservative management: retrospective review and literature survey. J Pediatr Surg. 2011;46(7):1425-31.

18. White M, Stella J. Ovarian torsion: 10-year perspective. Emerg Med Australas. 2005;17:231-7.

19. Sahlu Z, Negash S, Yusuf L. Adnexal torsion a fiveyears retrospective review in two hospitals. Ethiop Med J. 2014;52(4):155-64. 
20. Ryan MF, Desai BK. Ovarian torsion in a 5-Year old: a case report and review. Case reports in emergency medicine. 2012;2012(5):679121.

21. Poonai N, Poonai C, Lim R, Lynch T. Pediatric ovarian torsion: case series and review of the literature. Can J Surg. 2013;56(2):103-8.

22. Peng YY, Chen CP, Chien SC, Chen JR. Torsion of a huge ovarian cyst in a 90 -year-old woman. Taiwan J Obstet Gynecol. 2008;47(2):224-5.

23. Yousefi Z, Farazestanian M, Mottaghi M, Pourmoghadam N. Ovarian torsion in postmenopausal women and risk of malignancy. Journal of midwifery and reproductive health. 2015;3(4):479-82.

24. Huchon C, Fauconnier A. Adnexal torsion: a literature review. Eur J Obstet Gynecol Reprod Biol. 2010;150:8-12.

25. Melcer Y, Sarig-Meth T, Maymon R, Pansky M, Vaknin Z, Smorgick N. Similar but different: a comparison of adnexal torsion in pediatric, adolescent, and pregnant and reproductive-age women. J Womens Health (Larchmt). 2016;25(4):391-6.

26. Al-Turki HA. Adnexal torsion in children: the role of family practice physicians and paediatricians. J Taibah Uni Med Sci. 201;11(1):46-9.

27. Rousseau V, Massicot R, Dwarish AA, Sauvat F, Emond S, Thibaud E et al. Emergency management and conservative surgery of ovarian torsion in children: a report of 40 cases. J Pediatr Adolesc Gynecol. 2008;21:201-6.

28. Spinelli C, Buti I, Pucci V, Liserre J, Alberti E, Nencini L. Adnexal torsion in children and adolescents: new trends to conservative surgical approach -our experience and review of literature. J Gynecol Endocrinol. 2013;29(1):54-8.

29. Shadinger LL, Andreotti RF, Kurian RL. Preoperative sonographic and clinical characteristics as predictors of ovarian torsion. J Ultrasound Med. 2008;27:7-13.

30. Lee EJ, Kwon HC, Joo HJ, Suh JH, Fleischer AC. Diagnosis of ovarian torsion with color Doppler sonography: depiction of twisted vascular pedicle. J Ultrasound Med. 1998;17(2):83-9

31. Ben-Ami M, Perlitz Y, Haddad S. The effectiveness of spectral and color Doppler in predicting ovarian torsion: a prospective study. Eur J Obstet Gynecol Reprod Biol. 2002;104:64-6.

32. Albayram F, Hamper UM. Ovarian and adnexal torsion: spectrum of sonographic findings with pathologic correlation. J Ultrasound Med. 2001;20(10):1083-9.

33. Aziz D, Davis V, Allen L, Langer JC. Ovarian torsion in children: is oophorectomy necessary? J Pediatr Surg. 2004;39(5):750-3.

34. Tsafrir Z, Foad Azem, Hasson J, Solomon E, Almog $\mathrm{B}$, Nagar $\mathrm{H}$, et al. Risk factors, symptoms, and treatment of ovarian torsion in children: the twelve year experience of one center. J Minimal Invasive Gynaecol. 2012;19:29-33.

35. Lasso Betancor CE, Garrido Pérez JI, Murcia Pascual FJ, Granero Cendón R, Vargas Cruz V, Paredes Esteban RM. Ovarian torsion: long-term follow-up of the black-bluish ovary after laparoscopic detorsion. Cir Pediatr. 2014;27(1):2630 .

Cite this article as: Jain N, Manchanda R, Chithra S, Lekhi A. Adnexal torsion-symptoms, diagnosis and management: a review of literature. Int J Reprod Contracept Obstet Gynecol 2016;5:1276-84. 